\title{
Research and Realizing of a Data Acquisition and Storage System Based on ARM
}

\author{
Dongxing Wang ${ }^{1, a}$, Juan $\mathrm{Li}^{2, \mathrm{~b}}$ \\ ${ }^{1}$ Institute of Computer Science and Information Technology, Daqing Normal University, Daqing, \\ 163712, China \\ ${ }^{1}$ Institute of Computer Science and Information Technology, Daqing Normal University, Daqing, \\ 163712, China \\ aemail: cnwindy02@163.com, bemail:pang_hy@163.com
}

Keywords: ARM; data acquisition; SD card; embedded system; driver module

\begin{abstract}
Aim at solving the constraints of storage and processor of the slow data acquisition system, the system based on SD card and LPC2141 is designed. It adopts 6 channels AD sampling which sampling rate is more than $400 \mathrm{ks} / \mathrm{s}$ and SD card with large capacity storage, and extends the transmission function by using USB interface whose transmission rate is about $1 \mathrm{M} / \mathrm{s}$. The experimental results show that the system has good compatibility with different types of storage card and performs stably.
\end{abstract}

\section{Introduction}

With the development of embedded technology, the data acquisition system performance has a lot improved. The data acquisition system based on ARM has strong real-time and high accuracy characteristics. It has a lot of the data transmission modes and the transmission distance is long. The data storage of the system has the miniaturized and portable characteristics. It has become the mainstream by using the ARM processor to collect data which are the outgoing current and voltage signals or some thermodynamic parameters from the industrial field sensors [1]-[4].

The SD card is a kind of memory card with large capacity, high performance, small volume and simple access interface. The SD card is widely used in digital cameras, mobile phone and other mobile devices and has the advantages of low power consumption, nonvolatile and saving data without consuming energy etc.

So we predicted that the ARM data acquisition system based on the SD card will have a wide application prospect. Based on this, this paper designed a ARM data acquisition and storage system which use the SPI interface of LPC2141 chip (which is a kind of ARM chip) to drive SD card, and use the FAT file system to manage data. At the same time the hardware circuit of the system is designed, and made into the product. The testing results show that the system runs steadily and has good compatibility with different types of SD card.

\section{Structure design}

The system consists of data acquisition, data storage and data transmission, the framework of the whole system as shown in figure 1.

LPC2141 provides AD interface to receive the sensor analog input, and uses RS232 to communicate with the host computer which could send the commands to the host computer to control the system and change the system status. SD card stores digital data which has been converted from analog data by AD converter, and when the users get the data, the SD card could be taken out to read or the USB interface could be directly used to transport the data to the host computer [5]. According to the design, the system is divided into the following four relatively independent modules, and the hardware circuit and relevant software are independently designed. 


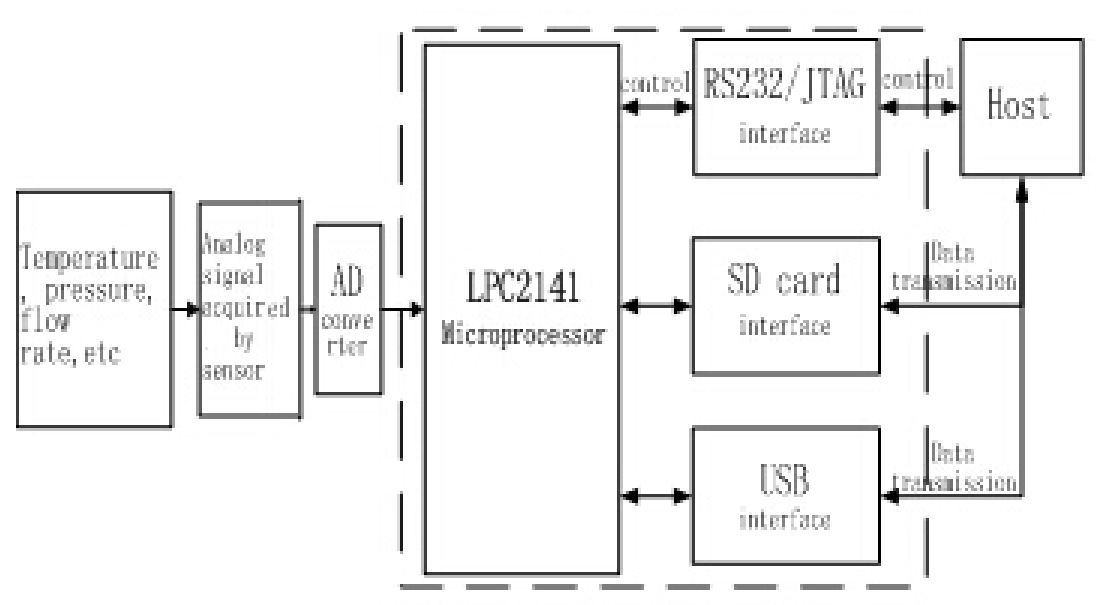

Fig.1. Structure diagram of data acquisition system

Module1: Minimum system module consists of LPC2141 chip, power supply, crystal, reset, JTAG connection circuit and so on.

Module2: AD module, consists of the pins associated with $\mathrm{AD}$ chip, the corresponding peripheral circuits of the reference voltage and so on.

Module3: USB module, consists of the pins associated with USB module and the corresponding peripheral circuit.

Module4: RS232 module, consists of the pins associated with UART module, SP3220 chip and the corresponding peripheral circuit.

\section{The main interface circuit design}

\subsection{AD interface circuit}

LPC2141 has a 10 bit, 6 channels AD converter, its reference voltage is provided by the VREF pin. The system gets the $3 \mathrm{~V}$ reference voltage by the resistor-divider principle.

At the same time, in order to improve sampling precision, the analog signal should firstly be filtered by the RC filter, and then enter the AD chip pin [6].

\subsection{RS232 interface circuit}

RS232 working voltage is $5 \mathrm{~V}$, and the system voltage is $3.3 \mathrm{~V}$, so we use the SP3220 chip to do the conversion. SP3220 is the conversion chip of RS232 working voltage, and it supports serial ISP (in system programmable) function. Short circuit ISP interface, When the system is reset and P0.14 port is in low level, the ISP hardware conditions are met and the chip is in the ISP state. When you want to use the ISP function, the system is connected to the PC with the serial port.

\subsection{SD card interface circuit}

The system uses the LPC2141 SPI function to provide the SD card interface. When the system is working, SD card is only needed to be inserted into socket, and then works.

The principle of the reading and writing the SD card is: the CMD signal line of the SD card is connected to the CPU CARD_MOSI interface, the master output signal, and the slave input signal. SD card DAT0 signal line is connected to the CPU CARD_MISO, which the SPI master input the signal, and the slave output the signal. The two signal lines are the data lines and are used to realize SPI reading and writing of the SD card. When the SD card is inserted, the detection signal line CARD-INSERT is pulled low by 11 pin of the socket. When the card is not inserted, CARD-INSERT is at high level. CARD-WP used to detect whether the card is write protected. When the card is inserted, it is write-protected if CARD-WP outputs high level, or is not write-protected.

\subsection{USB interface circuit}

The LPC2141 has a built-in USB device controller; you can use the USB interface to communicate. In order to make the USB driver software more flexibly control the connection between the USB device and the host computer, the circuit uses P0.31 to realize the UP_LED 
function. When the device is configured, it outputs low level, USB_LED is lightening up. When the device is not configured or is suspended, it outputs high level, and USB_LED is extinguished. The P0.23 pin is used to detect whether the USB bus is inserted into the pin. Because of being the self power supply system, the pin can be directly connected with the VBUS.

\section{Implementation and testing}

\subsection{The system implementation}

The PCB of the test circuit board has four layer boards and the panel size is $10 \mathrm{~cm} x 8 \mathrm{~cm}$ in design. USB, SD card, AD input pins and serial interface and the related circuitry are located at the different positions to eliminate interference and be convenient to upgrade and develop the new module for the system.

\subsection{SD card module test}

The two kind cards of SanDisk 2GB and Kingston 4GB are used to test the large capacity card compatibility of the system.

The specific method is: Editing program, and setting a cache INT8U sdbuf [2048] used to access the data in the card. To describe the differences comprehensively between large amounts and a small amount of data in program for cards to process, we read and write $64 \mathrm{~KB}, 256 \mathrm{~Kb}, 1024 \mathrm{~KB}$, $4096 \mathrm{~kb}$ size files respectively to the two cards, realized through the functions of the $\mathrm{f}$ _mount, f_open, f_read, f_write, f_close in the ff.c.

\subsection{Results analysis of the experiment}

Through the integration test of the system, and the data transmission performance test for the SD card module, the data shows that the different models of SD card write the file faster when the file is bigger. The whole test results show that the system works stable, have good compatibility with different SD cards, and can storage data for long time.

There are shortcomings found during the test. In order to improve the performance of the system, the next version can improve from the following aspects:

1. Design the lithium battery power supply circuit to improve the system's portability.

2. Considering the hardware resource constraints of the embedded system, optimize the code of some certain functions [7].

\section{Conclusions}

On the basis of ARM technology, design and realize a data acquisition system with serial communication and large capacity storage performance which solve the constraints of hardware resource such as memory and processor. By use of the rich hardware resource and strong control function of the ARM chip, a data acquisition system with the large storage capacity is realized which can collect and preserve the data for a long time. At the same time, the USB interface with the PC connection is designed to transmit data conveniently.

Compared to the similar products, the system improves the data storage performance and has the good application value.

\section{Acknowledgement}

The studies were funded by Youth Fund Project of Daqing Normal University (12ZR21).

\section{References}

[1]FAN Sheng-yi. The principle of ARM and embedded systems combat [M].Beijing: China Machine Press, 2007.

[2]HAN Shan, GUO Yun, FU Hai-yan. The technology explain and example analysis of ARM application development [M]. Beijing: Tsinghua University Press, 2007. 
[3]DING Feng, LU Li, XU Jing. The development of ARM system from practice to improvement[M]. Beijing: China Electric Power Press, 2007.

[4]WU Fei, LI Yang-jun. The data acquisition system of sensor network based on S3C2410 [J]. Shanxi Electronic Technology, 2010, 37 (4): :17-18.

[5]WANG Xiao-rong,HE Xiao-song, ZHU Yong-rong. Hardware design of data acquisition system based on ARM Processor[J]. Microcomputer information, 2008, 24(8-2):161-162,199.

[6]PENG Min. Design of data acquisition systems based on embedded system [J]. Information technology, 2010,33 (9) : 88-89, 92.

[7] LIU Yu, MENG Xiao-hua. Design and implementation of communication module in smart home terminal based on embedded system [J]. Computer Engineering and Design, 2010, 31(8):1689-1692. 